\title{
Micro-morphological Characterization of Fresh Cannabis sativa L. Roots After Lead Exposure Using Low Temperature Method for ESEM
}

\author{
Vilém Neděla1 ${ }^{\text {, Eva Tihlaříková1, Biljana Đorđević }}{ }^{2}$ Nikola Slámová2 \\ 1. Environmental Electron Microscopy Group, Institute of Scientific Instruments of the Czech Academy \\ of Sciences, Brno, Czech Republic \\ 2. Department of Plant Biology, Mendel University in Brno, Brno, Czech Republic
}

Cannabis sativa L. is an important multi-purpose herbaceous plant known for its high content of phytochemicals but as well as, an important source of both cellulosic and woody fibers. Heavy metal accumulation in plants is often restricted to the root tissue, with only small amounts transported to the shoot [1]. An understanding of the toxicological and physiological responses of a plant to metal contamination is of particular relevance when attempting to predict the impact of a metal ion on the growth of an industrial plant like hemp. This is particularly important nowadays when the number of contaminated riparian ecosystems is increasing. Heavy metal exposure can affect plant's capability to receive nutrients and change plant's anatomy. There are various methods to determine morphological changes in root cross sections. However, there are disadvantages of these methods such as limited resolution (histology) [2], time consuming chemical treatment with possible artifacts formation (SEM) and high cost of the equipment (cryo-SEM) [3].

An optimal way is to study plant samples as close as possible to their native state in environmental scanning electron microscope (ESEM). The ESEM observation of fully wet samples is usually affected with radiation damage and lower resolution; this can be overcome by the use of special methods like the Low Temperature Method for the ESEM (LTM) [4].

For this study, industrial hemp variety Bialobrzeskie obtained from Agritec Plant Research Institute Ltd., Šumperk, Czech Republic was selected. For obtaining plantlets for in vitro propagation, hemp seeds were firstly thoroughly washed with few drops of detergent under running tap water and afterwards surface sterilized with $0.2 \%$ of mercury chloride for 13 minutes and rinsed three times with sterile distilled water. The sterilized seeds were germinated on nutritional medium. The seeds were maintained in a cultivation room under 18/6 light dark cycle at $24 \pm 2{ }^{\circ} \mathrm{C}$ for 15 days. Five shoot tips ( $\sim 2 \mathrm{~cm}$ in length) were placed equidistantly to each other in a jar on nutritional media. Standard cultivation medium was used as a control, and experimental variant was supplemented with $\mathrm{Pb}^{2+}$ at 750 $\mu \mathrm{M}$ concentration. Stock solution of $\mathrm{Pb}^{2+}$ was prepared by mixing $\mathrm{Pb}\left(\mathrm{NO}_{3}\right)_{2}$ with ethylene diamine tetraacetic acid (EDTA) in a 1:1 molar ratio and stirring at $50{ }^{\circ} \mathrm{C}$ for $1 \mathrm{~h}$. The filter-sterilized Pb-EDTA complex was added to the autoclaved culture medium to avoid precipitation. Plantlets were grown on nutritional media for one month until roots were developed and used for ESEM observation.

Roots of the hemp were cut to approximately $2 \mathrm{~mm}$ long segments and placed into a drop of $2 \mu \mathrm{lof}$ water to obtain better thermal contact between the sample and the Peltier stage (PS). In ESEM observation, the temperature of the cooled PS was $2.5^{\circ} \mathrm{C}$, vapor pressure was around $730 \mathrm{~Pa}$ to obtain $100 \%$ relative humidity. Conditions for the LTM method were adjusted according to [4], the water vapor pressure was $150 \mathrm{~Pa}$ and the temperature of the cooled PS was $-20{ }^{\circ} \mathrm{C}$. All experiments were performed on FEI ESEM QUANTA 650FEG and carried out under constant operating conditions. The beam accelerating voltage of $10 \mathrm{kV}$, probe current of $40 \mathrm{pA}$ and working distance of $8.8 \mathrm{~mm}$ were used. 
Fig. 1 shows comparison between the results of wet observation in ESEM and using the LTM. Samples are imaged in their fully wet state (Fig. 1A, B), nevertheless sample surface is covered with thin water layer preventing observation of the surface microstructure. Gradual evaporation of water makes partial visibility of the surface possible (Fig. 1B), however, further water evaporation causes a sample collapse (Fig. 1C). Application of the LTM allows decreasing amount of water on the sample surface and exposing its microstructure (Fig. 1D, E). Our results revealed that the LTM is capable of indication of root firmness caused by different composition of cultivation medium. Plantlets growing in medium enriched with $\mathrm{Pb}^{2+}$, evince higher cell wall density and can be observed with no shrinkage (Fig. 1D). Untreated control samples were very soft and the application of the LTM causes cell collapse (Fig. 1E).

Results show that the LTM is fast and inexpensive method well applicable on commercial ESEM. Newly it was shown that is also suitable for preparation of cross sections of the samples designated for the study of plant anatomy that is impossible in fully wet state. When the LTM is used on the samples with thin cell wall, its application is limited. Nevertheless, after advanced adjustment of the LTM according to the sample specificity, it enables to obtain very good results (Fig. 1E). No chemical treatment and no presence of ice allow to use the LTM also as a preparation method for EDS analysis $[5]$.

\section{References:}

[1] GJ Taylor, Journal of Plant Nutrition 10 (1987), p. 1213.

[2] M Vaculik et al, Environmental Pollution 163 (2012), p. 117.

[3] V Neděla et al, Micron 84 (2016), p. 67.

[4] V Neděla et al, Microscopy Research and Technique 78 (2015), p. 13.

[5] The authors acknowledge funding from the MEYS CR (LO1212), its infrastructure by the MEYS CR and the EC (CZ.1.05/2.1.00/01.0017) and by the CAS (RVO:68081731) and Internal Grant Agency (IGA) Faculty of AgriSciences MENDELU No. TP 5/2017.

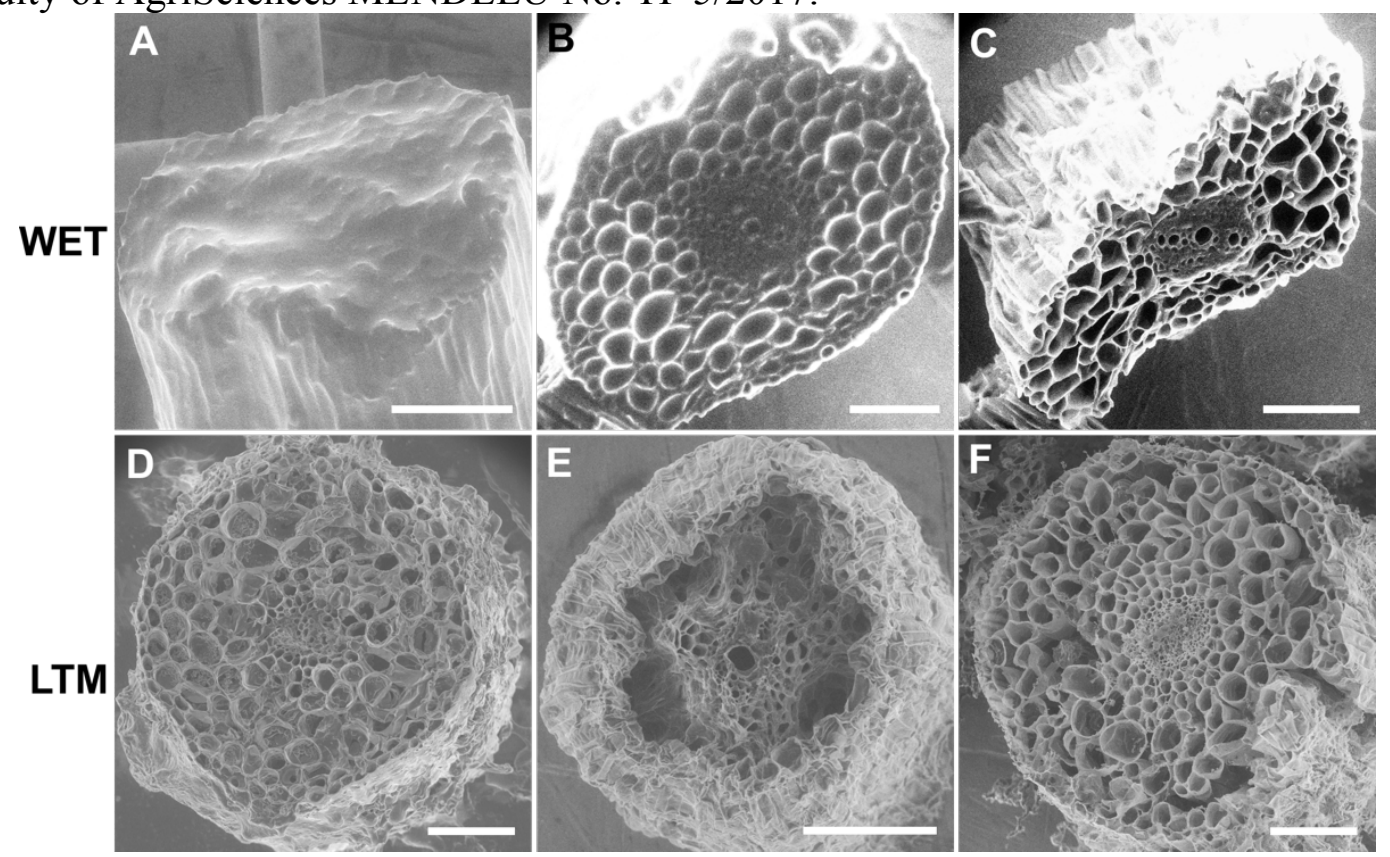

Figure 1. Cross sections of hemp root observed using ESEM in wet state (A, B, C) and using LTM for $\operatorname{ESEM}(\mathrm{C}, \mathrm{D}, \mathrm{E})$. The sample with affected with $\mathrm{Pb}$ evinces higher cell wall density (D) unlike the control sample (E). For observation of control samples, the LTM had to be adjusted (F). Bar $100 \mu \mathrm{m}$. 